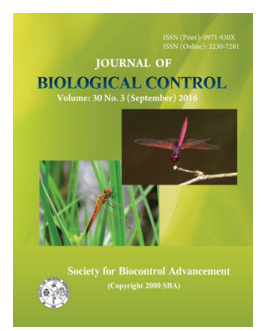

\title{
Non-target effects of insecticides on the abundance of predators in Soybean
}

\author{
A. A. MOTAPHALE ${ }^{*}$, B. B. BHOSLE and B. B. GAIKWAD \\ Department of Agricultural Entomology, Vasantrao Naik Marathwada Krishi Vidyapeeth, Parbhani - 431402, Maharashtra, India \\ ${ }^{*}$ Corresponding author E-mail: dr.ashvini24@gmail.com
}

\begin{abstract}
Highest population of Coccinella septempunctata after first spray was observed in untreated control(1.92) followed by Bt(1.70), Beauveria bassiana (1.66) whereas during second spray more number of C. septempunctata observed in untreated control (2.01) followed by Azadirachtin $1500 \mathrm{ppm}$ (1.77) and NSKE 5\% (1.74). The population of Chrysoperla arabica was highest in untreated control (1.47) followed by NSKE 5\% (0.99) and Beauveria bassiana (0.96) during first spray and after second spray observed in untreated control (1.30) followed by Beauveria bassiana (0.94) and Nomuraea rileyi $(0.93)$.
\end{abstract}

KEY WORDS: Coccinella septempunctata, Chrysoperla arabica, natural enemies, NSKE, Beauveria bassiana

(Article chronicle: Recived: 18-09-2016; Revised: 24-09-2016; Accepted: 27-09-2016))

\section{INTRODUCTION}

Soybean (Glycine max (L.) Merill) grows well in varied agro-climatic conditions and emerged as important commercial crop in many countries and international trade of soybean has been spreading globally. The use of broadspectrum insecticides is one of the main obstacles to effective biological control because natural enemies are just as susceptible to the insecticide as the pest. Different pesticides have been used for control of soybean pests. But several pesticides have broad spectrum activity and are highly toxic to non-target organisms. Among many predators on soybean like lady bird beetle (Coccinella septempunctata) and chrysopa (Chrysoperla arabica) are highly predominant. Most species of ladybirds are considered beneficial because they are predators of Homoptera or Acarina, many of which are considered to be pests. A single lady beetle may consume as many as 5,000 aphids in its lifetime. Releases of C. carnea eggs in field cages at rates of 50,000 and 100,000 per acre also provided effective control of the tobacco budworm reported by Ridgway, et al., (1969). Therefore, present field studies were undertaken to determine the effects of selected insecticides and biorationals on population of $C$. septempunctata) and C. arabica) on soybean.

\section{MATERIAL AND METHODS}

Experiments were conducted during kharif 2010-2011 and 2011-12, in the field of Department of Agricultural Entomology, College of Agriculture, Marathwada Krishi
Vidyapeeth, Parbhani (Maharashtra). Totally two sprayings were made 30 and 55 days after sowing respectively. Population of C. septempunctata and C. arabica were recorded on randomly selected five plants from each net plot on weekly interval during both the years. Yield of soybean from each net plot was recorded separately and computed on hectare basis.

\section{Statistical analysis}

As per Gomez and Gomez (1984), the data on larval population were subjected to $\sqrt{x}+0.5$ transformation i.e. Poisson formula, whereas data on per cent infestation were transformed into arc sin transformation values before statistical analysis.
$\mathrm{X}=$ average number of pest population. The trans formed data was analyzed through ANOVA

\section{RESULT AND DISCUSSION}

\section{Effect of insecticides and biorationals on population of Coccinella septempunctata}

First spraying (2010-11): The data in respect of population of $C$. septempunctata) on soybean after the first spray are presented in Table 1 revealed that the significantly highest population was observed in untreated control (2.00) followed by Beauveria bassiana (1.97) and Nomuraea rileyi (1.91) found at par with each other. Next best treatment was $B t$ (1.75) followed by diflubenzuron $25 \mathrm{WP}$ (1.67), Buprofezin (1.11), NSKE 5\% (1.07), and Azadirachtin 1500 
ppm (1.03). Among the insecticides maximum number of C. septempunctata was observed in indoxacarb $14.5 \%$ (0.99) followed by emamectin benzoate $5 \mathrm{SG}(0.98)$, chlorpyriphos 20 EC (0.88), spinosad 45SC (0.80), Quinalphos $25 \mathrm{EC}(0.75)$ and rynaxypyr $20 \mathrm{SC}(0.60)$.

First spraying (2011-12): The significantly highest population of Coccinella septempunctata was observed in untreated control (1.85) followed by azadirachtin $1500 \mathrm{ppm}$ (1.80), Bt (1.65LB/5plants), buprofezin (1.60) found at par with each other. Next best treatment was $N$. rileyi (1.40) followed by Beauveria bassiana (1.35), Diflubenzuron 25 WP (1.32), NSKE 5\% (1.17). Among the insecticides maximum number was observed in Spinosad 45SC (1.00) followed by Indoxacarb 14.5\% (0.92), Chlorpyriphos $20 \mathrm{EC}$ (0.88), Rynaxypyr $20 \mathrm{SC}(0.79)$, Emamectin benzoate 5SG (0.78) and Quinalphos 25 EC (0.78) found at par with each other. Pooled data : Significantly highest population was observed in untreated control (1.92) followed by Bt (1.70), Beauveria bassiana (1.66), Nomuraea rileyi (1.65), Diflubenzuron 25 WP (1.49), Azadirachtin 1500 ppm (1.41), Buprofezin (1.35) and NSKE 5\% (1.12) found at par with each other. Maximum number of Coccinella septempunctata among the insecticides was observed in Indoxacarb $14.5 \%$ (0.95) followed by Spinosad 45SC (0.90), Emamectin benzoate 5SG (0.88), Quinalphos 25 EC (0.83), Chlorpyriphos $20 \mathrm{EC} \mathrm{(0.81)} \mathrm{and} \mathrm{Rynaxypyr} 20 \mathrm{SC}$ (0.69).

Second spraying (2010-11) : The data in respect of population of Coccinella septempunctata on soybean after the first spray are presented in Table 1 revealed that the significantly highest population was observed in untreated control (2.02) followed by Azadirachtin $1500 \mathrm{ppm}$ (1.77), NSKE 5 \% (1.68), Bt (1.42), Buprofezin (1.40) Diflubenzuron 25 WP (1.07), Beauveria bassiana (1.00) and Nomuraea rileyi (1.00). Among the insecticides maximum number was observed in Spinosad 45SC (1.00) followed by Quinalphos 25 EC (0.96), Chlorpyriphos 20 EC (0.95), Emamectin benzoate 5SG (0.93), Indoxacarb $14.5 \%(0.92)$ and Rynaxypyr 20 SC (0.89). Second spraying (201112): The significantly highest population was observed in untreated control (2.00) followed by NSKE 5\% (1.80), Azadirachtin 1500 ppm (1.77), Nomuraea rileyi (1.69), Bt (1.64), Beauveria bassiana (1.34), Buprofezin (1.33) and Diflubenzuron 25 WP (1.29). Among the insecticides maximum number of Coccinella septempunctata was in Emamectin benzoate 5SG (1.00) followed by Indoxacarb 14.5\% (0.97), Spinosad 45SC (0.96), Quinalphos 25 EC (0.92), Chlorpyriphos 20 EC (0.89) and Rynaxypyr 20 SC (0.84). Pooled data: presented in Table 1 showed that the significantly highest population was observed in untreated control (2.01) followed by Azadirachtin 1500 ppm (1.77), NSKE
5\% (1.74), Bt (1.53), Buprofezin (1.36) Nomuraea rileyi (1.34) Diflubenzuron 25 WP (1.18) Beauveria bassiana (1.17). Among the insecticides maximum number of Coccinella septempunctata was observed in Spinosad 45SC (0.98) followed by Emamectin benzoate 5SG (0.96), Indoxacarb 14.5\% (0.94), Quinalphos 25 EC (0.92) followed by Chlorpyriphos 20 EC (0.93), Rynaxypyr 20 SC (0.86).

Results obtained during present experiment are in conformity with the findings of Patil et al. (2004) who reported that seed dressing with acetamiprid @ $17 \mathrm{~g}, 26 \mathrm{~g}$ and 25 $\mathrm{g}$ a.i/ha and imidacloprid @ $10 \mathrm{~g}$ a.i/ha were toxic to coccinellids and spiders whereas imidacloprid was more toxic than acetamiprid Patil \& pawar (1994) studied the effect of chemicals and biopesticides on lady bird beetles. They observed highest population in untreated control plot followed by NPV@500 and 250 LE/ha. They also reported that the endosulfan@350 g a.i./ha and cypermethrin@60 $\mathrm{g}$ a.i/ha were toxic to lady bird beetles per five plant were lowest after 3 days of spray, which however increased after 7 and 14 days.

\section{Effect of insecticides and biorationals on population of} Chrysoperla arabica after first spraying

First spraying (2010-11): The data in respect of population of Chrysoperla arabica on soybean after the first spray are presented in Table 2 which revealed that the significantly highest population was observed in untreated control (1.60) followed by Buprofezin (1.00), NSKE 5\% (1.00), Beauveria bassiana (0.93), Nomuraea rileyi (0.88), Diflubenzuron 25 WP (0.78), Bt (0.75) and Azadirachtin 1500 ppm (0.65) significantly superior over rest of the treatments. Maximum number of Chrysopa among the insecticides was found in Chlorpyriphos $20 \mathrm{EC}(0.23)$ followed by Spinosad 45SC (0.32), Quinalphos 25 EC (0.34), Emamectin benzoate $5 \mathrm{SG}(0.22)$ and no population was observed in Indoxacarb $14.5 \%$ and Rynaxypyr 20 SC. First spraying (2011-12): the significantly highest population of Chrysopa was observed in untreated control (1.35) followed by Nomuraea rileyi (1.00), Beauveria bassiana (1.00), NSKE 5\% (0.98), Bt (0.94), Diflubenzuron 25 WP (0.81), Azadirachtin 1500 ppm (0.78) and Buprofezin (0.75) and were found at par with each other and significantly superior over rest of the treatments. Maximum number of Chrysopa among the insecticides was found in Emamectin benzoate 5SG (0.75) followed by Spinosad 45SC (0.63), Indoxacarb $14.5 \%$ (0.55), Rynaxypyr 20 SC (0.40), Quinalphos 25 EC (0.30) and Chlorpyriphos 20 EC (0.20). Pooled data: the significantly highest population of Chrysoperla arabica was observed in untreated control (1.47) followed by NSKE 5\% (0.99), Beauveria bassiana (0.96), Nomuraea rileyi (0.94), Buprofezin (0.87), Bt (0.84), Diflubenzuron 25 WP (0.79) 
Table 1. Effect of insecticides and biorationals on population of Lady beetle after first and second spraying

\begin{tabular}{|c|c|c|c|c|c|c|c|c|}
\hline \multirow{2}{*}{$\begin{array}{l}\text { Sr. } \\
\text { No. }\end{array}$} & \multirow{2}{*}{ Treatments } & \multirow{2}{*}{ Dose / lit. } & \multicolumn{3}{|c|}{ First spraying } & \multicolumn{3}{|c|}{ Second spraying } \\
\hline & & & 2010 & 2011 & Pooled & 2010 & 2011 & Pooled \\
\hline 1. & Buprofezin25 SC@2ml & $2 \mathrm{ml}$ & $\begin{array}{c}1.11 \\
(1.42)\end{array}$ & $\begin{array}{c}1.60 \\
(1.44)\end{array}$ & $\begin{array}{c}1.35 \\
(1.41)\end{array}$ & $\begin{array}{c}1.40 \\
(1.37)\end{array}$ & $\begin{array}{c}1.33 \\
(1.35)\end{array}$ & $\begin{array}{c}1.36 \\
(1.38)\end{array}$ \\
\hline 2. & Diflubenzuron25 WP@0.8g & $0.8 \mathrm{~g}$ & $\begin{array}{c}1.67 \\
(1.23)\end{array}$ & $\begin{array}{c}1.32 \\
(1.34)\end{array}$ & $\begin{array}{c}1.49 \\
(1.31)\end{array}$ & $\begin{array}{c}1.07 \\
(1.24)\end{array}$ & $\begin{array}{c}1.29 \\
(1.33)\end{array}$ & $\begin{array}{c}1.18 \\
(1.28)\end{array}$ \\
\hline 3. & Azadirachtin 1500 ppm@2ml & $2 \mathrm{ml}$ & $\begin{array}{c}1.03 \\
(1.25)\end{array}$ & $\begin{array}{c}1.80 \\
(1.51)\end{array}$ & $\begin{array}{c}1.41 \\
(1.30)\end{array}$ & $\begin{array}{c}1.77 \\
(1.52)\end{array}$ & $\begin{array}{c}1.77 \\
(1.60)\end{array}$ & $\begin{array}{c}1.77 \\
(1.55)\end{array}$ \\
\hline 4. & NSKE 5\%@1ml & $1 \mathrm{ml}$ & $\begin{array}{c}1.07 \\
(1.55)\end{array}$ & $\begin{array}{c}1.17 \\
(1.29)\end{array}$ & $\begin{array}{c}1.12 \\
(1.41)\end{array}$ & $\begin{array}{c}1.68 \\
(1.44)\end{array}$ & $\begin{array}{c}1.80 \\
(1.47)\end{array}$ & $\begin{array}{c}1.74 \\
(1.64)\end{array}$ \\
\hline 5. & Nomuraearileyi@4g & $4 \mathrm{~g}$ & $\begin{array}{c}1.91 \\
(1.57)\end{array}$ & $\begin{array}{c}1.40 \\
(1.37)\end{array}$ & $\begin{array}{c}1.65 \\
(1.42)\end{array}$ & $\begin{array}{c}1.00 \\
(1.23)\end{array}$ & $\begin{array}{c}1.69 \\
(1.35)\end{array}$ & $\begin{array}{c}1.34 \\
(1.35)\end{array}$ \\
\hline 6. & Beauveriabassiana@4g & $4 \mathrm{~g}$ & $\begin{array}{c}1.97 \\
(1.49)\end{array}$ & $\begin{array}{c}1.35 \\
(1.35)\end{array}$ & $\begin{array}{c}1.66 \\
(1.41)\end{array}$ & $\begin{array}{c}1.00 \\
(1.22)\end{array}$ & $\begin{array}{c}1.34 \\
(1.47)\end{array}$ & $\begin{array}{c}1.17 \\
(1.29)\end{array}$ \\
\hline 7. & Bt 5\%@100g/ha & $100 \mathrm{~g} / \mathrm{ha}$. & $\begin{array}{c}1.75 \\
(1.21)\end{array}$ & $\begin{array}{c}1.65 \\
(1.46)\end{array}$ & $\begin{array}{c}1.70 \\
(1.31)\end{array}$ & $\begin{array}{c}1.42 \\
(1.39)\end{array}$ & $\begin{array}{c}1.64 \\
(1.22)\end{array}$ & $\begin{array}{c}1.53 \\
(1.43)\end{array}$ \\
\hline 8. & Emamectin benzoate $5 \mathrm{SG} @ 0.4 \mathrm{~g}$ & $0.4 \mathrm{~g}$ & $\begin{array}{c}0.98 \\
(1.13)\end{array}$ & $\begin{array}{c}0.78 \\
(1.12)\end{array}$ & $\begin{array}{c}0.88 \\
(1.12)\end{array}$ & $\begin{array}{c}0.93 \\
(1.18)\end{array}$ & $\begin{array}{c}1.00 \\
(1.20)\end{array}$ & $\begin{array}{c}0.96 \\
(1.13)\end{array}$ \\
\hline 9. & Spinosad 45\%@0.4ml & $0.4 \mathrm{ml}$ & $\begin{array}{c}0.80 \\
(1.21)\end{array}$ & $\begin{array}{c}1.00 \\
(1.22)\end{array}$ & $\begin{array}{c}0.90 \\
(1.20)\end{array}$ & $\begin{array}{c}1.00 \\
(1.21)\end{array}$ & $\begin{array}{c}0.96 \\
(1.21)\end{array}$ & $\begin{array}{c}0.98 \\
(1.21)\end{array}$ \\
\hline 10. & Indoxacarb 14.5\%@1ml & $1 \mathrm{ml}$ & $\begin{array}{c}0.99 \\
(1.04)\end{array}$ & $\begin{array}{c}0.92 \\
(1.19)\end{array}$ & $\begin{array}{c}0.95 \\
(1.11)\end{array}$ & $\begin{array}{c}0.92 \\
(1.18)\end{array}$ & $\begin{array}{c}0.97 \\
(1.15)\end{array}$ & $\begin{array}{c}0.94 \\
(1.19)\end{array}$ \\
\hline 11. & Rynaxypyr 20 SC@0.3ml & $0.3 \mathrm{ml}$ & $\begin{array}{c}0.60 \\
(1.11)\end{array}$ & $\begin{array}{c}0.79 \\
(1.13)\end{array}$ & $\begin{array}{c}0.69 \\
(1.14)\end{array}$ & $\begin{array}{c}0.89 \\
(1.19)\end{array}$ & $\begin{array}{c}0.84 \\
(1.18)\end{array}$ & $\begin{array}{c}0.86 \\
(1.16)\end{array}$ \\
\hline 12. & Chlorpyriphos 20SC@2ml & $2 \mathrm{ml}$ & $\begin{array}{c}0.75 \\
(1.25)\end{array}$ & $\begin{array}{c}0.88 \\
(1.17)\end{array}$ & $\begin{array}{c}0.81 \\
(1.18)\end{array}$ & $\begin{array}{c}0.95 \\
(1.15)\end{array}$ & $\begin{array}{c}0.92 \\
(1.15)\end{array}$ & $\begin{array}{c}0.93 \\
(1.17)\end{array}$ \\
\hline 13. & Quinalphos 25 EC@2ml & $2 \mathrm{ml}$ & $\begin{array}{c}0.88 \\
(1.38)\end{array}$ & $\begin{array}{c}0.78 \\
(1.13)\end{array}$ & $\begin{array}{c}0.83 \\
(1.28)\end{array}$ & $\begin{array}{c}0.96 \\
(1.15)\end{array}$ & $\begin{array}{c}0.89 \\
(1.18)\end{array}$ & $\begin{array}{c}0.92 \\
(1.15)\end{array}$ \\
\hline 14. & Untreated control & - & $\begin{array}{c}2.00 \\
(1.54)\end{array}$ & $\begin{array}{c}1.85 \\
(1.53)\end{array}$ & $\begin{array}{c}1.92 \\
(1.47)\end{array}$ & $\begin{array}{c}2.02 \\
(1.56)\end{array}$ & $\begin{array}{c}2.00 \\
(1.58)\end{array}$ & $\begin{array}{c}2.01 \\
(1.59)\end{array}$ \\
\hline & $\mathrm{SE} \pm$ & - & 0.04 & 0.04 & 0.06 & 0.03 & 0.09 & 0.05 \\
\hline & $\mathrm{CD}$ at $P=5 \%$ & - & 0.11 & 0.11 & 0.13 & 0.09 & 0.28 & 0.17 \\
\hline
\end{tabular}

and Azadirachtin 1500 ppm (0.75) significantly superior over rest of the treatrments. Maximum number of Chrysoperla arabica among the insecticides was found in Emamectin benzoate 5SG (0.48) followed by Spinosad 45SC (0.47), Quinalphos 25 EC (0.32) followed by Indoxacarb $14.5 \%$ (0.27), Chlorpyriphos 20 EC (0.21) and Rynaxypyr $20 \mathrm{SC}(0.20)$.

Second spraying (2010-11): The data in respect of population of Chrysoperla arabica on soybean after the second spray are presented in Table 2 revealed that the significantly highest population was observed in untreated control (1.40) followed by Diflubenzuron 25 WP (1.00) Beauveria bassiana (1.00), Nomuraea rileyi (0.90), Bt (0.85), Buprofezin (0.79), Azadirachtin 1500 ppm (0.74) and NSKE 5\% (0.65). Maximum number among the insecticides was found in Spinosad 45SC (0.35) followed by Emamectin benzoate 5SG (0.18) Indoxacarb 14.5\% (0.15) and zero population was noticed in Quinalphos 25 EC, Rynaxypyr 20 SC and Chlorpyriphos 20 EC. Second spraying (2011-12):
The significantly highest population Chrysoperla arabica was observed in untreated control (1.20) followed by $B t$ (1.00), Nomuraea rileyi (0.96), NSKE 5\% (0.94) Beauveria bassiana (0.88), Diflubenzuron 25 WP (0.80), Azadirachtin 1500 ppm (0.79) and Buprofezin (0.68). Maximum number of Chrysopa among the insecticides was found in Indoxacarb 14.5\% (0.25) Rynaxypyr 20 SC (0.22), Quinalphos 25 EC (0.20), Chlorpyriphos 20 EC (0.18) and zero population was noticed in Emamectin benzoate 5SG and Spinosad 45SC. Pooled data: showed that the significantly highest population of Chrysopa was observed in untreated control (1.30) followed by Beauveria bassiana (0.94), Nomuraea rileyi (0.93), Bt (0.92), Diflubenzuron $25 \mathrm{WP}$ (0.90), NSKE 5\% (0.79), Azadirachtin 1500 ppm (0.76) and Buprofezin $(0.73)$ found significantly superior over rest of the treatments. Maximum number of Chrysopa among the insecticides was found in Indoxacarb 14.5\% (0.20) followed by Spinosad 45SC (0.17), Rynaxypyr 20 SC (0.11), Quinalphos 25 EC (0.10), Emamectin benzoate 5SG and Chlorpyriphos $20 \mathrm{EC}(0.09)$ Results obtained during present experi- 
ment are in conformity with the findings of Sarode and Sonalkar (1999) who evaluated the insecticidal products for their biosafety to Chrysoperla zotswani Arabica in the laboratory. None of the insecticides were found to be safer to the predator. A. J.Varenhorst et al.,(2012) reported that selective insecticides should affect the target insect although having minimal effects on the natural enemy community that is present in the held during insecticide application; they should effectively control the pest, and conserve the natural enemy population.

Effect of insecticides and biorationals on yield of soybean $(\mathrm{kg} / \mathrm{ha})$

The data in respect of yield of soybean during kharif
2010-11, 2011-12 and pool analyzed are presented in Table 2. During kharif 2010-11, the significantly maximum yield of soybean was recorded in Rynaxypyr $20 \mathrm{SC}(2518 \mathrm{~kg} / \mathrm{ha})$ followed by Spinosad 45SC (2400 kg/ha), Emamectin benzoate $5 \mathrm{SG}(2300 \mathrm{~kg} / \mathrm{ha})$ were found at par with each other. The next best treatment was Indoxacarb $14.5 \%(2181 \mathrm{~kg} / \mathrm{ha})$ followed by Quinalphos 25 EC (2018 kg/ha) and Chlorpyriphos $20 \mathrm{EC}(2009 \mathrm{~kg} / \mathrm{ha})$ were significantly superior over untreated control and at par with each other. Significantly minimum yield was observed in untreated control (1375 $\mathrm{kg} / \mathrm{ha}$ ). During kharif 2011-12, the significantly maximum yield of soybean was recorded in Rynaxypyr 20 SC (2368 $\mathrm{kg} / \mathrm{ha}$ ) followed by Spinosad 45\% (2370 kg/ha), Emamectin benzoate $5 \mathrm{SG}(2319 \mathrm{~kg} / \mathrm{ha})$, Chlorpyriphos 20 EC (2019 kg/

Table 2. Effect of insecticides and biorationals on population of Chrysopa after first and second spraying and grain yield

\begin{tabular}{|c|c|c|c|c|c|c|c|c|c|c|c|}
\hline \multirow{2}{*}{$\begin{array}{l}\text { Sr. } \\
\text { No. }\end{array}$} & \multirow{2}{*}{ Treatments } & \multirow{2}{*}{ Dose / lit. } & \multicolumn{3}{|c|}{ First spraying } & \multicolumn{3}{|c|}{ Second spraying } & \multicolumn{3}{|c|}{ Average grain yield } \\
\hline & & & 2010 & 2011 & Pooled & 2010 & 2011 & Pooled & 2010 & 2011 & Pooled \\
\hline 1. & Buprofezin25 SC@2ml & $2 \mathrm{ml}$ & $\begin{array}{c}1.00 \\
(1.22)\end{array}$ & $\begin{array}{c}0.75 \\
(1.22)\end{array}$ & $\begin{array}{c}0.87 \\
(1.21)\end{array}$ & $\begin{array}{c}0.79 \\
(1.13)\end{array}$ & $\begin{array}{c}0.68 \\
(1.08)\end{array}$ & $\begin{array}{c}0.73 \\
(1.10)\end{array}$ & 1820 & 1789 & 1804 \\
\hline 2 & Diflubenzuron25 WP@0.8g & $0.8 \mathrm{~g}$ & $\begin{array}{c}0.78 \\
(1.13)\end{array}$ & $\begin{array}{c}0.81 \\
(1.14)\end{array}$ & $\begin{array}{c}0.79 \\
(1.13)\end{array}$ & $\begin{array}{c}1.00 \\
(1.22)\end{array}$ & $\begin{array}{c}0.80 \\
(1.13)\end{array}$ & $\begin{array}{c}0.90 \\
(1.18)\end{array}$ & 1900 & 1880 & 1890 \\
\hline 3 & Azadirachtin 1500 ppm@2ml & $2 \mathrm{ml}$ & $\begin{array}{c}0.65 \\
(1.07)\end{array}$ & $\begin{array}{c}0.78 \\
(1.12)\end{array}$ & $\begin{array}{c}0.75 \\
(1.09)\end{array}$ & $\begin{array}{c}0.74 \\
(1.11)\end{array}$ & $\begin{array}{c}0.79 \\
(1.13)\end{array}$ & $\begin{array}{c}0.76 \\
(1.12)\end{array}$ & 1792 & 1794 & 1793 \\
\hline 4 & NSKE 5\%@1ml & $1 \mathrm{ml}$ & $\begin{array}{c}1.00 \\
(1.22)\end{array}$ & $\begin{array}{c}0.98 \\
(1.21)\end{array}$ & $\begin{array}{c}0.99 \\
(1.21)\end{array}$ & $\begin{array}{c}0.65 \\
(1.07)\end{array}$ & $\begin{array}{c}0.94 \\
(1.30)\end{array}$ & $\begin{array}{c}0.79 \\
(1.20)\end{array}$ & 1730 & 1696 & 1713 \\
\hline 5 & Nomuraea rileyi@4g & $4 \mathrm{~g}$ & $\begin{array}{c}0.88 \\
(1.17)\end{array}$ & $\begin{array}{c}1.00 \\
(1.22)\end{array}$ & $\begin{array}{c}0.94 \\
(1.19)\end{array}$ & $\begin{array}{c}0.90 \\
(1.16)\end{array}$ & $\begin{array}{c}0.96 \\
(1.20)\end{array}$ & $\begin{array}{c}0.93 \\
(1.17)\end{array}$ & 1715 & 1795 & 1755 \\
\hline 6 & Beauveriabassiana@4g & $4 \mathrm{~g}$ & $\begin{array}{c}0.93 \\
(1.29)\end{array}$ & $\begin{array}{c}1.00 \\
(1.22)\end{array}$ & $\begin{array}{c}0.96 \\
(1.25)\end{array}$ & $\begin{array}{c}1.00 \\
(1.22)\end{array}$ & $\begin{array}{c}0.88 \\
(1.17)\end{array}$ & $\begin{array}{c}0.94 \\
(1.19)\end{array}$ & 1797 & 1770 & 1783 \\
\hline 7 & Bt 5\%@100g/ha & $100 \mathrm{~g} / \mathrm{ha}$. & $\begin{array}{c}0.75 \\
(1.11)\end{array}$ & $\begin{array}{c}0.94 \\
(1.23)\end{array}$ & $\begin{array}{c}0.84 \\
(1.17)\end{array}$ & $\begin{array}{c}0.85 \\
(1.16)\end{array}$ & $\begin{array}{c}1.00 \\
(1.02)\end{array}$ & $\begin{array}{c}0.92 \\
(1.20)\end{array}$ & 1800 & 1765 & 1782 \\
\hline 8 & Emamectin benzoate $5 \mathrm{SG} @ 0.4 \mathrm{~g}$ & $0.4 \mathrm{~g}$ & $\begin{array}{c}0.22 \\
(0.84)\end{array}$ & $\begin{array}{c}0.75 \\
(1.13)\end{array}$ & $\begin{array}{c}0.48 \\
(0.48)\end{array}$ & $\begin{array}{c}0.18 \\
(0.82)\end{array}$ & $\begin{array}{c}0.00 \\
(0.70)\end{array}$ & $\begin{array}{c}0.09 \\
(0.75)\end{array}$ & 2300 & 2319 & 2309 \\
\hline 9 & Spinosad 45\%@0.4ml & $0.4 \mathrm{ml}$ & $\begin{array}{c}0.32 \\
(0.90)\end{array}$ & $\begin{array}{c}0.63 \\
(1.02)\end{array}$ & $\begin{array}{c}0.47 \\
(0.96)\end{array}$ & $\begin{array}{c}0.35 \\
(0.91)\end{array}$ & $\begin{array}{c}0.00 \\
(0.70)\end{array}$ & $\begin{array}{c}0.17 \\
(0.81)\end{array}$ & 2400 & 2370 & 2385 \\
\hline 10 & Indoxacarb 14.5\%@1ml & $1 \mathrm{ml}$ & $\begin{array}{c}0 \\
(0.70)\end{array}$ & $\begin{array}{c}0.55 \\
(1.09)\end{array}$ & $\begin{array}{c}0.27 \\
(0.89)\end{array}$ & $\begin{array}{c}0.15 \\
(0.98)\end{array}$ & $\begin{array}{c}0.25 \\
(0.86)\end{array}$ & $\begin{array}{c}0.20 \\
(0.91)\end{array}$ & 2181 & 2000 & 2090 \\
\hline 11 & Rynaxypyr 20 SC@0.3ml & $0.3 \mathrm{ml}$ & $\begin{array}{c}0 \\
(0.70)\end{array}$ & $\begin{array}{c}0.40 \\
(0.90)\end{array}$ & $\begin{array}{c}0.20 \\
(0.80)\end{array}$ & $\begin{array}{c}0.00 \\
(0.70)\end{array}$ & $\begin{array}{c}0.22 \\
(0.84)\end{array}$ & $\begin{array}{c}0.11 \\
(0.75)\end{array}$ & 2518 & 2368 & 2443 \\
\hline 12 & Chlorpyriphos 20SC@2ml & $2 \mathrm{ml}$ & $\begin{array}{c}0.23 \\
(0.85)\end{array}$ & $\begin{array}{c}0.20 \\
(0.90)\end{array}$ & $\begin{array}{c}0.21 \\
(0.87)\end{array}$ & $\begin{array}{c}0.00 \\
(0.70)\end{array}$ & $\begin{array}{c}0.18 \\
(0.81)\end{array}$ & $\begin{array}{c}0.09 \\
(0.73)\end{array}$ & 2009 & 2019 & 2014 \\
\hline 13 & Quinalphos25 EC@2ml & $2 \mathrm{ml}$ & $\begin{array}{c}0.34 \\
(1.84)\end{array}$ & $\begin{array}{c}0.30 \\
(0.91)\end{array}$ & $\begin{array}{c}0.32 \\
(0.87)\end{array}$ & $\begin{array}{c}0.00 \\
(0.70)\end{array}$ & $\begin{array}{c}0.20 \\
(0.83)\end{array}$ & $\begin{array}{c}0.10 \\
(0.75)\end{array}$ & 2018 & 1919 & 1968 \\
\hline 14 & Untreated control & - & $\begin{array}{c}1.60 \\
(1.44)\end{array}$ & $\begin{array}{c}1.35 \\
(1.35)\end{array}$ & $\begin{array}{c}1.47 \\
(1.39)\end{array}$ & $\begin{array}{c}1.40 \\
(1.37)\end{array}$ & $\begin{array}{c}1.20 \\
(1.30)\end{array}$ & $\begin{array}{c}1.30 \\
(1.31)\end{array}$ & 1375 & 1497 & 1436 \\
\hline & $\mathrm{SE} \pm$ & - & 0.04 & 0.06 & 0.06 & 0.04 & 0.05 & 0.05 & 80 & 70 & 55 \\
\hline & $\mathrm{CD}$ at $P=5 \%$ & - & 0.13 & 0.17 & 0.19 & 0.12 & 0.15 & 0.16 & 234 & 204 & 103 \\
\hline
\end{tabular}


ha) were significantly superior over untreated control. Significantly minimum yield was observed in untreated control $(1497 \mathrm{~kg} / \mathrm{ha})$. The pooled data presented in Table 2 showed that significantly maximum yield of soybean was recorded in Rynaxypyr $20 \mathrm{SC}$ (2443 kg/ha) followed by Spinosad $45 \mathrm{SC}$ (2385 kg/ha), Emamectin benzoate $5 \mathrm{SG}(2309 \mathrm{~kg} / \mathrm{ha})$ were found at par with each other. The next best treatment was Indoxacarb $14.5 \%(2090 \mathrm{~kg} / \mathrm{ha})$ and Chlorpyriphos 20 EC (2014 kg/ha) were significantly superior over untreated control. Significantly minimum yield was observed in untreated control $(1436 \mathrm{~kg} / \mathrm{ha})$. The present findings are also agreement with those of Taware et al.(2000) who also recorded highest seed yield with chlorpyriphos spray @ 1.5 $\mathrm{kg}$ a.i. /ha at 8-10 DAG (3548 kg/ha) followed by thaimethoxam seed treatment @3 g and 5 g/kg and phorate10 G@ $10 \mathrm{~kg} / \mathrm{ha}$ as soil application before sowing. Virkar (2004) also recorded highest yield of soybean was obtained from the plots treated with chlorpyriphos 20 EC @ 1.51/ha (1396 $\mathrm{kg} / \mathrm{ha})$ followed by triazophos 40 EC @ 0.8 1/ha (1269 kg/ ha).

\section{References}

Varenhorst J, O'Neal ME. 2012. The response of natural enemies to selective insecticides applied to soybean. Environ Entomol. 41 (6): 1565-1574. Doi: 10.1603/ EN12068
Patil SB, Pawar VM. 1994. Bioefficacy and compatibility of methomyl $40 \mathrm{SP}$ alone and in combination with HaNPV against coccinellid predator of cotton. Pestol. 18(10): 25-26.

Patil RH, Kulkarni KA. 2004. New promising seed dress for the management of soybean seedling borers. Pestol. 28(3): 22-25.

Ridgway RL, Jones SL. 1969. Inundative releases of Chrysopa carnea for control of Heliothis on cotton. DOI: http://dx.doi.org/10.1093/jee/62.1.177 177-180. First published online: 1 February 1969

Sarode SV, Sonalkar VD. 1999. Ovicidal effect of some insecticides against Chrysoperla carnea. Steph Pesticide Res J. 11(1): 97-98.

Tawre SP, Varghese P, Halvankar GB, Raut VM. 2003. Chemical control of leaf miner (Aproaerema modicella) of soybean (Glycine max.). Indian J Agric Sci. 73(2): 124-125.

Virkar AR. 2004. Studies on chemical control and economics of major insect pests of soybean (Glycine max L.). M.Sc. (Agri.) thesis, MAU, Parbhani. 